\title{
Pre-to-post race changes in self-reported depression scores in ultra-distance triathletes - a pilot study
}

\author{
A M Harrison (MD) ${ }^{1}$ \\ D T Yaldoo (PhD)' \\ C M Fiesler (MD) $)^{2}$ \\ J T Connor (MS) ${ }^{3}$
}

'Division of Pediatrics

${ }^{2}$ Department of Orthopedics and Sports Medicine

${ }^{3}$ Department of Epidemiology and Biostatistics, Cleveland Clinic Foundation, Cleveland, Ohio, USA

\begin{abstract}
Objective. To describe the effect of participation in an Ultra-distance triathlon on self-reported depression.

Design and interventions. This was a prospective pre and post-race observational study. Triathletes were eligible to participate if they planned to participate in an ultra. distance triathlon between June and December 2001. Thiry-eight triathletes were enrolled, and 33 completed the study. Questionnaires and the Beck Depression Index (BDI-H) were completed approximately 4 weeks before and 2 weeks after an ultra-distance triathlon.

Main outcome measures. The primary outcome was change in BDI-II score from pre- to post race. Secondary outcomes included effect of demographic variables, experience with ultra-distance trathlons, and attainment of pre-race goals.

Results. The study sample ineluded 19 males $(58 \%)$ and 14 females $(42 \%)$. Nineteen triathietes $(58 \%)$ were participating in their first ultra-distance triathlon, while $14(42 \%)$ had previously completed one. The 33 triathletes competed in 9 different races seven $(21 \%)$ had previously been diagnosed with and treated for a mood disorder, while 4 $(12 \%)$ were receiving treatment at the time of their race. BDI II scores did not change significantly from pre-to post-race, with an average decrease of $1.36(95 \% \mathrm{Cl}-0.1$ -29). $p=0.07$. Novice ultra-distance trathletes expert enced an average decrese of $2.9(95 \% \mathrm{Cl} .1 .3-4.6$ $p=0.0005$ ) in depression score. Changes in BDIII scores were not associated with race completion, mood
\end{abstract}

\section{CORRESPONDENCE:}

\section{A M Harrison}

9500 Euclid Avenue, S-10A

Cleveland, $\mathrm{OH} 44195$

Tel: 216-444 9370

Fax: 216-444 3310

E-mail: harrism1@ccf.org disorder, or post-race injury.

Conclusions. No significant decline in mood was found in this sample of ultra-distance triathletes.

\section{Introduction}

Since the first Hawaii Ironman (IM) Triathion in 1978, participation in ultra-distance triathlons $(3.8 \mathrm{~km}$ swim, $180 \mathrm{~km}$ cycle, and $42.2 \mathrm{~km}$ run) has grown tremendously to over 15000 participants and 35 ultra-distance races each year ${ }^{5.33}$ Although some professional athletes compete in these races, the vast majority of the participants in ultra-distance triathlons are recreational athletes. However as more citizen athletes become involved in extreme sporting challenges, it will be incumbent on health providers to thoroughly understand the positive and negative physical and psychoactive effects of exercise.

In moderation, exercise can improve physical health. ${ }^{12,14}$ Similarly, mood state can be measurably altered by bouts of exercise. ${ }^{16,22}$ Exercise has been successfully incorporated into the treatment regimen of patients with depression and anxiety, with good results. ${ }^{22}$ Intense and/or prolonged training for endurance sports without adequate recovery may result in impaired performance, termed overtraining. .7.18 $^{\text {Overtraining }}$ may result in mood disturbances that improve following a period of rest and recovery. ${ }^{15}$ Physicians should be as facile at identifying exercise as an aetiology of pathology as they are at prescribing it to prevent or treat disease.

Some information exists regarding the relationship between intense or prolonged aerobic exercise and selfreported mood. Post-race depression has been associated with poor race performance in age-group and elite distance runners. ${ }^{13,19}$ Novice, previously unfit runners who ran a marathon after a 7-month training programme experienced a heterogeneous response, ranging from feeling 'drained' and 'burned out' post-race to highly motivated. ${ }^{26}$ Furthermore, a study of 106 male runners who completed the 1989 Stockholm marathon with finishing times between 3 hours and 3 hours and 45 minutes found that participation in a marathon race affected mood in a negative manner. ${ }^{6}$ This effect was short-lived, with rapid return to baseline mood. Similar data are not available for triathletes. 
An ultra-marathon is defined by runners as any event longer than the standard $42.2 \mathrm{~km}$ marathon. Ultra-marathoners have baseline personality characteristics that are significantly less anxious, hostile, and depressed than those of control subjects. ${ }^{10}$ A case study of a female ultra-marathoner who completed a 48-hour race described a slight increase in seif-reported depression 3 days after the event." Her mood had recovered to pre-race status by the final data point 1 month after the race. A prospective study of 56 male ultramarathoners found minor increases in self-reported depression 1 hour after successfully completing a 50 or 100 mile running race. ${ }^{31}$ Triathletes reserve the term ultra-distance for races equal to or exceeding the Ironman in distance.

Anecdotal descriptions of depressed mood following ultradistance triathlons have been reported in the lay press, on Internet list-serves, and by personal communication. Many triathletes refer to this phenomenon as 'post-Ironman depression.' Dave Scott, former professional triathlete and six-time Hawaii IM winner, states, 'Everyone who has finished an Ironman recognises the inherent psychological let-down after the event.' Scott employs the terms 'malaise', 'distraught', 'despair', and 'cavern of disorder' to describe post-race mood. ${ }^{28}$

A Medine search of relevant key words did not reveal any references that describe acute changes in mood state associated with participation in any triathlon, let alone an ultraendurance length event. We hypothesised that athletes competing in an ultra-distance triathlon would experience a mild, yet significant, post-race depression, as measured by the Beck Depression Inventory, second edition (BDI-II). ${ }^{2}$

\section{Methods}

Subjects were recruited via triathlon websites on the Internet. A request for subjects was posted on the tri-drs listserve (a discussion forum devoted to the exchange of triathlon-related information), the IM USA bulletin board (a discussion forum for participants in ultra-distance triathlons), and an ultra-distance triathlon race promotion web site. ${ }^{8,9,32}$ Triathletes were considered eligible to participate in our study if they planned to complete an ultra-distance triathlon within the 6-month period from June through December 2001. No compensation was offered for participation. They were promised results of the study once it underwent peer review.

Subjects were assigned a number, which was the only identifier in the database. Basic demographic data, contact information, and date of the triathlon were collected. Six weeks prior to the goal race, a packet containing two copies of the informed consent document (one to return and one to retain), a detailed pre-race questionnaire, a copy of the BDI-II, and two stamped pre-addressed return envelopes were mailed to each subject. One envelope was for the informed consent document, while the other was for the anonymous return mailing of the questionnaire and $\mathrm{BDI}-\mathrm{II}$. The pre-race questionnaire inquired about age, sex, marital status, education, employment, triathlon participation history, training practices, race goals, past and current concerns about mood disturbance, and past and current treatment for mood disturbance. Subjects were instructed to complete and return all information 4 weeks prior to their goal race, which is when ultra-distance triathletes are typically at peak training volume.
During the week of the goal race, a post-race packet containing a detailed post-race questionnaire, a copy of the BDI$\mathrm{II}$, and a stamped pre-addressed return envelope was mailed to the subject. The subject was instructed to complete and return all information 2 weeks after the race. The post-race questionnaire inquired about race performance, satisfaction with race performance, weather conditions during the race, plans for future races, current concerns about mood disturbance, and current treatments for mood disturbance.

The BDI-II is a 21-item self-report measure used to assess the presence and severity of depression within the preceding 2 weeks, consistent with the diagnostic criteria outlined in the American Psychiatric Association's Diagnostic and Statistical Manual of Mental Disorders (Fourth edition). ${ }^{1,2}$ This tool is among the most widely used measures of depression and has been validated among clinical and non-clinical populations. ${ }^{2}$ Scores on the BDI-II range from 0 to 13 for normal subjects, 14 - 19 for mild depression, 20 - 28 for moderate depression, and up to a maximum of 63 for a severely depressed person. ${ }^{2}$ The mean change in BDI-Il score as a function of exercise is $0.5 .^{21}$

The primary outcome was change in BDI-II score from pre to post race. Measures of central tendency are described as mean ( $95 \%$ confidence interval $(\mathrm{Cl})$ of the mean). Categorical variables are described as frequencies (\%). A paired $t$-test was used to assess the primary outcome. It was determined that 24 participants would offer $90 \%$ power using a paired $t$-test with a 0.01 type I error rate to detect a mean change of one-point from pre-to-post race, assuming the standard deviation (SD) in the change in BDI-II scores would be 1.0. All eligible subjects who responded to our calls for subjects were enrolled. Enrolling more than 24 subjects ensured adequate power if the BDI-II SDs were larger than expected or if subject follow-up was difficult to achieve.

Multivariate linear regression analysis was used as a secondary analysis to simultaneously measure the effect of covariates such as gender, triathlon experience, attainment of pre- and post-race goals, remaining injury free, and race completion on the change in pre- to post-race BDI-Il scores. Statistical significance was reached if $p<0.01$. Because other outcomes were investigated and due to the exploratory nature of this secondary analysis, a smaller alpha level was chosen to preserve the type I error rate.

\section{Ethical considerations}

The Cleveland Clinic Foundation Institutional Review Board formally exempted our study.

\section{Results \\ Cohort demographics}

Forty-seven triathletes contacted us. Thirty-eight were prospectively enrolled in this study, and $33(87 \%)$ completed the study. The study sample included 19 males $(58 \%)$ and 14 females (42\%). Twenty-two (67\%) were married, $7(21 \%)$ were single, $3(9 \%)$ were divorced, and $1(3 \%)$ was separated. Fifteen triathletes (45\%) had children. Fifteen $(45 \%)$ had a college/university degree as a terminal degree, while 18 (55\%) had an advanced degree. Nineteen triathletes (58\%) were participating in their first ultra-distance triathlon, and the 
other $14(42 \%)$ had experience ranging from 1 to 6 ultra-distance triathlons. No participant was a professional triathlete.

The 33 triathletes competed in 9 different races. Sixteen racers (48\%) competed in IM USA, and $10(30 \%)$ competed in IM Canada. One triathlete competed in each of the following races: the 5430 Triathlon, the Great Floridian Triathlon, IM Austria, IM Florida, IM Hawaii, IM Switzerland, and the Mohican Pineman. Thirty participants $(91 \%)$ completed their triathlon. The 3 triathletes $(9 \%)$ who did not complete their race identified 'stomach problems' and 'dehydration' as the primary reason for failing to finish the goal race. Twentyseven triathletes $(82 \%)$ claim to have met their primary prerace goal. 'Finishing' was the primary goal for $25(76 \%)$ of the triathletes. 'Having fun' and staying 'injury free' were also common goals. All 33 triathletes reported on the post-race questionnaire that they planned to participate in another ultra-distance triathlon.

\section{Previous mood concerns}

Thirteen of the triathletes (39\%) reported previous concerns about their mood. Seven (21\%) had previously been diagnosed with and treated for a mood disorder, 6 for depression $(18 \%)$, and 1 for panic disorder (3\%). A summary of these athletes is shown in Table I. Past therapies included medication $(N=1,3 \%)$, psychotherapy $(N=2,6 \%)$, and a combination of psychotherapy and medication $(N=4,12 \%)$. Four triathletes (12\%) were being treated for a mood disorder at the time of their race. Two $(6 \%)$ were taking medication and undergoing psychotherapy, while $2(6 \%)$ were being treated with medication only.

TABLE L. Subject characteristics vs, prior mood disorder (summary statistics are median (IQR) or percentage).

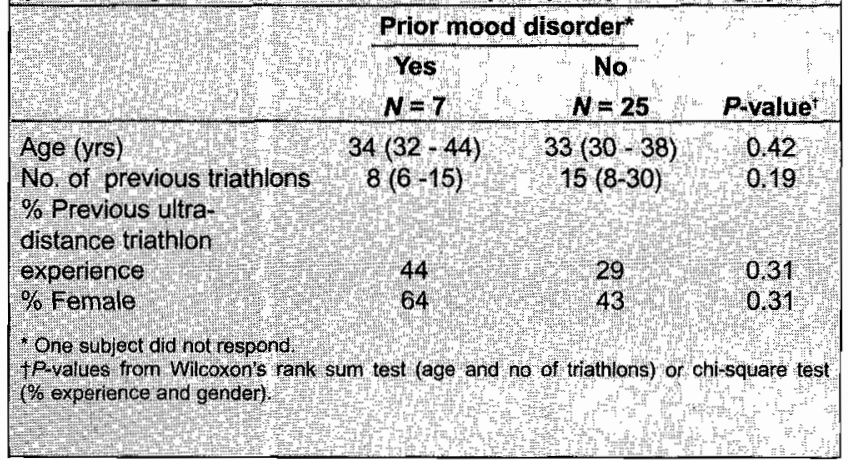

\section{Primary outcome: pre-to-post race change in BDI-II scores}

Pre-race and post-race BDI-II scores are shown in Table II. The majority of triathletes scored in the non-depressed range (i.e. 'normal range') both pre- and post-race. Fig. 1 shows the distribution of BDI-II scores pre- and post-race and the distribution of the pre-to-post race changes in BDI-II scores. The mean pre-race BDI-II score is 6.8 (95\% Cl: $4.9-8.7)$. The mean post-race BDI-II score is $5.4(95 \% \mathrm{Cl}: 3.5-7.3)$. The mean pre-to-post race change was 1.36 units $(95 \% \mathrm{Cl}:-0.1$ 2.9), an average decrease in depression score post-race. Both the pre- and post-race means are clearly in the non-

\begin{tabular}{|c|c|c|c|c|}
\hline $\begin{array}{l}\text { TABLE II. I } \\
\text { by ultra-dis }\end{array}$ & to-post & e cha & n depre & \\
\hline $\begin{array}{l}\text { Preto post } \\
\text { race BD/1/ } \\
\text { score } \\
\text { change }\end{array}$ & $\begin{array}{l}\text { No vitra- } \\
\text { distance } \\
\text { triathlon } \\
\text { experience }\end{array}$ & Per cent & $\begin{array}{l}\text { Ultra- } \\
\text { distance } \\
\text { trathion } \\
\text { experience }\end{array}$ & Per cent \\
\hline Decrea & $10 / 19$ & 53 & $5 / 14$ & 36 \\
\hline & $8 / 19$ & 42 & $3 / 14$ & 21 \\
\hline Inc & 119 & 5 & $6 / 14$ & 43 \\
\hline Mean change & 2.95 & $p=0.0005$ & 0.79 & $p=0.43$ \\
\hline & & & & \\
\hline
\end{tabular}

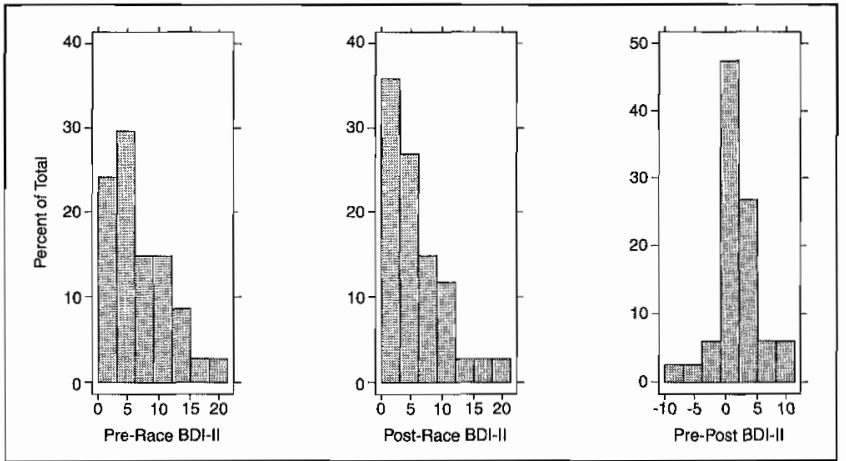

Fig. 1. Histograms of pre-race BDI-II scores (left), post-race BDI-II scores (middle), and pre-to-post race change scores (right). A positive value in the right hand panel indicates a lower, less depressed, post-race BDI-II score. The majority of athletes had a heightening of mood post-race.

depressed range. The study's primary question, viz. whether there is an average change in ultra-distance triathlete BDI-II scores pre-to-post race, is tested using a paired $t$-test showing $p=0.07$. While post-race BDI-II scores decreased approximately twice as often as they increased, $15(45 \%)$ of the triathletes' BDI-II scores decreased pre-to-post race, 11 (33\%) remained the same, and $7(21 \%)$ increased, there is not sufficient evidence to indicate a mean change in BDI-II scores from pre-to-post race.

The SD in score changes, 4.2 , was higher than projected during the study's design. This resulted in an appreciably lower power than expected $(40 \%)$ even with the larger than projected sample size.

A second paired $t$-test was also performed excluding the 4 patients who were being treated for a mood disorder at the time of their race. This paired $t$-test of the 29 patients not being treated for mood disorder also indicates no significant change in mood pre-to-post race. Mean BDI-II scores decreased from 6.7 to 5.6 , a mean change of 1.1 units $(95 \%$ $\mathrm{Cl}:-0.55$ to $2.69, p=0.18$ ).

\section{Secondary outcomes: sub-group analysis}

There is no predictable pre- to post-race change in BDI-II scores in this sample of ultra-distance triathletes. There are, however, notable differences in depression changes between experienced and inexperienced competitors and between genders.

Novice triathletes $(N=19)$ experienced an average 2.95 unit $(95 \% \mathrm{Cl}: 1.3-4.6, p=0.0005)$ decrease in BDI-II scores 
pre-to-post race, from a mean of 6.79 to 3.84 . Triathletes who had previously competed in 1 or more ultra-distance races had a mean increase of 0.8 units ( $95 \% \mathrm{Cl}:-1.2-2.7, p=0.4)$, from a mean of 6.8 to 7.6. Table II shows that over one-half of all novice triathletes reported an improvement in mood post-race. One novice triathlete $(5 \%)$ was measurably more depressed post-race. Experienced ultra-distance triathletes were roughly equally distributed between increased, no change, and decreased depression scores. Comparison of the changes between groups indicates that inexperienced triathletes clearly have a healthier mean decrease in BDI-II score (i.e. improvement in mood) than experienced triathletes $(p=0.004)$.

This experience effect is partially confounded by gender. Females showed a mean decrease in depression pre-to-post race of 2.4 ( $95 \% \mathrm{Cl}: 0.24-4.48, p=0.03$ ), while the decrease shown by males was insignificant $(0.63,95 \% \mathrm{Cl}$ : $-1.19-2.45$, $p=0.5$ ). Table Ill shows the change in depression scores by gender. Men's scores increase, decrease, and remain the same with nearly equal frequency. The majority of women's scores decrease post-race. The difference between genders, however, is not statistically significant $(p=0.25)$.

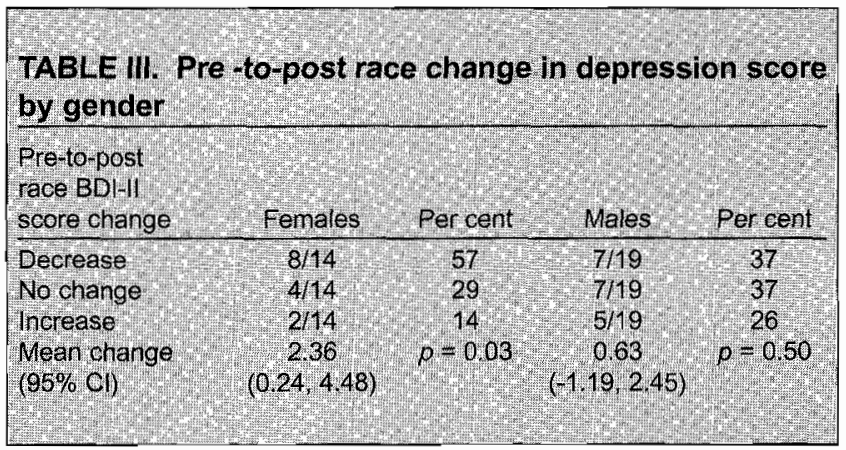

Thirty participants $(91 \%)$ completed their triathlons. The mean decrease in BDI-II scores for those who completed their race is 1.4 units. The mean decrease for the $3(9 \%)$ who did not complete their race is 1.0 unit. No formal statistical test was performed since few participants failed to finish their race.

Likewise there is no meaningful difference between preand post-race BDI-II score changes based on post-race injury or illness. Average BDI-Hl decrease for the 10 triathletes (33\%) citing injury or illness in the 2 weeks after their race is 1.3 units. Average BDI-II decrease for the 23 non-injured or ill athletes is 1.4 units. Mean difference is 0.1 units $(95 \% \mathrm{Cl}:-3.4$ 3.2, $p=0.96$ ).

While there were too few triathletes with previous mood disorders to allow for a reasonably powered statistical test, the 4 triathletes receiving treatment for a mood disorder at the time of their triathlon demonstrated larger decreases in mean $\mathrm{BDI}-\mathrm{Il}$ scores than the remaining 29 triathletes. For the 4 triathletes who reported an active mood disorder, mean change was a decrease of $3.5(95 \% \mathrm{Cl}:-0.5-7.5)$. For the other 29 triathletes, mean change was a 1.1 unit increase (95\% Cl: $-0.4-2.5$, range -10 to $11, p=0.26$ ).

\section{Multivariate analysis}

Although experience level is somewhat confounded by gender
- inexperienced triathletes are equally split between females and males (10 vs. 9 ), but $70 \%$ of experienced triathletes in this study are male - we can conclude that experience, not gender, is the primary predictor of change in BDI-Il scores pre-topost race. According to $\mathrm{R}^{2}$ values, experience explains $20 \%$ of the variability in the change in BDI-II scores, whereas gender explains only $4 \%$ of the variability in the change in BDI-II scores. Similarly, when testing if experience or gender is the key predictor of change in BDl-Il score, Schwarz's Bayesian Information Criteria indicates that there is over $99.99 \%$ probability that including experience alone provides the optimal model. ${ }^{27}$

Twenty-one different symptom areas are assessed using the BDI-II. Notable post-race changes - seen by decreases in BDI-II component scores - were fewer guilty feelings $(p=0.01)$ and increased interest in sex $(p=0.03)$. It was also common to report less energy after the race than before. Fig. 2 highlights the changes in individual BDI-II components pre-topost race.

The only statistical comparison that achieves our predetermined $p<0.01$ cutoff is that inexperienced triathletes see a greater decrease in $\mathrm{BDI}-\mathrm{II}$ scores than experienced triathletes.

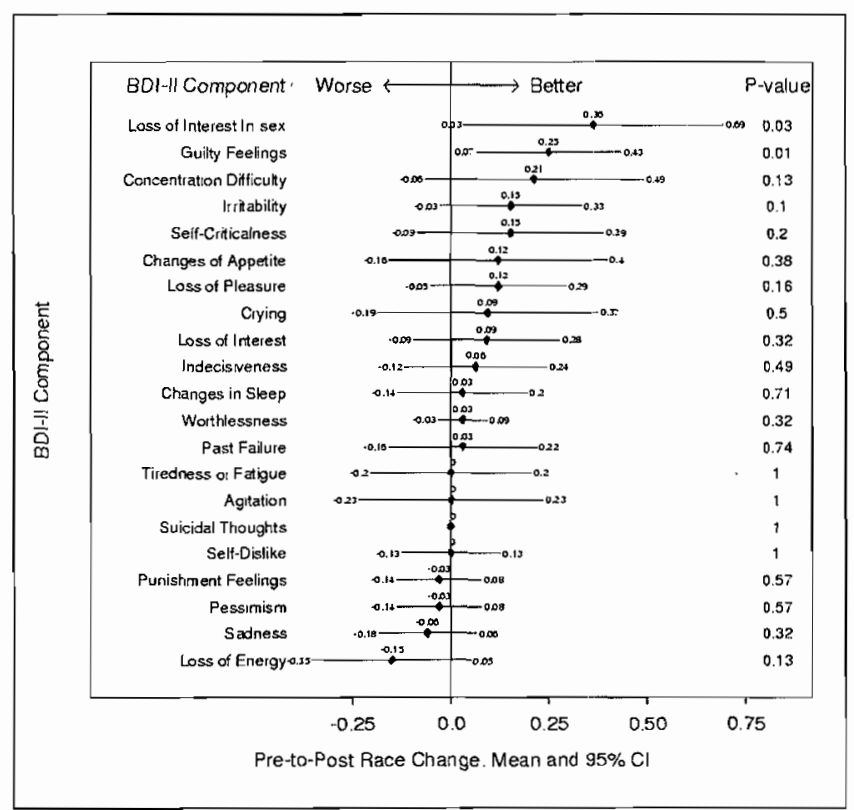

Fig. 2. Means and $95 \%$ confidence intervals for changes in the 21 BDI-II component scores pre-to-post race. Positive changes indicate lower, less depressed, post-race BDI-II scores.

\section{Discussion}

The BDI-II did not detect a change in self-reported depression scores in triathletes participating in an ultra-distance triathlon. The majority of participants scored in the non-depressed range both pre- and post-race, and the average change in BDI-II score was statistically and clinically non-significant. A plurality of experienced triathletes $(43 \%)$ reported a decline in mood post-race, consistent with anecdotal reports, although this finding was not statistically significant.

Analyses of subsets of this sample revealed interesting trends. Gender trends suggest that women may be more 
likely than men to experience some improvement in mood post-race; notably, this gender effect appears to be confounded by experience. First-time competitors reported a statistically significant improvement in mood post-race, and first-time competitor status was found to be the primary predictor of improvement in mood pre-to-post race. This phenomenon may be related to feelings of relief after a period of notable pre-race worry among novices, as well as feelings of accomplishment after completing this physically and mentally challenging endeavour. A series of additional post-race measures of mood may be useful in future research, to assess whether the improvement in mood reported by novice triathletes persists over time.

No meaningful difference was found between pre- and post- race BDI-II scores relative to completion of race or postrace injury or illness. Previous mood disorder did not correlate with mood change pre-to-post race. The trend toward improved mood from pre- to post- race in triathletes with previous mood disorder deserves further investigation in a study with adequate statistical power to examine this sub-population. Similarly, only $18 \%$ of subjects failed to meet their prerace goals. Further studies with a larger sample size may be able to identify whether this cognitive attribution contributes to change in mood.

The decision to utilise the BDI-II as the depression measure for this study posed some potential limitations. The BDIII is designed to measure severity of depression through the assessment of symptoms corresponding to DSM-IV criteria for diagnosing depressive disorders, although it has been validated among clinical and non-clinical populations. ${ }^{1,2}$ Given that most participants scored in the non-depressed range both preand post-race, it may have been useful if a measure that was more sensitive to subtle changes in mood in a non-clinical population had been used. Also of note, results should be interpreted carefully, as self-report inventories are subject to response bias; individuals may endorse more symptoms than they indeed experience or may minimise symptoms. While direct psychological evaluation by a professional might be considered as an alternative to reliance on self-report as a measure of change in mood, this would be costly and time intensive and may not be feasible. Barring direct examination by a professional, future survey studies should attempt to identify subjects' life stressors apart from sport.

Thirty-nine per cent of respondents reported either treatment for a mood disorder or concern about their mood. Approximately $9.5 \%$ of North American adults experience depression each year. ${ }^{24}$ The epidemiology of mood disorders and other psychological issues in triathletes is not known. At this time, it is not possible to know whether the subjects of our pilot study are representative of other age-group triathletes.

Our self-selected group may not be representative in other ways. As subjects were recruited over the Internet, access to this technology and a modicum of computer literacy may have introduced selection bias. The group of triathletes represented in this study reported a higher degree of depression than is common in the population, and one must wonder if individuals with past or current mood concerns had greater interest in this study and were therefore more likely to volunteer for enrollment. Also of note, all of our subjects were at least college educated. Since statistics regarding educational attainment for North American triathletes are not published, this is a potential source of bias. Based on our limited enrollment of subjects, one must consider our work a pilot study. Although the number of triathletes participating in ultra-distance races has increased, they are scattered over a very wide geographic area. This dispersion raises challenges regarding recruitment of large numbers of subjects into a longitudinal, prospective trial, as well as precise timing of BDI-II administration.

Subsequent studies should evaluate whether our timing of BDI-II administration was optimal. We chose to make a measurement 4 weeks pre-race in order to avoid the possibility of pre-race anxiety affecting BDI-II score. It is possible that a score obtained at another time point would be more representative of pre-race mood. The pre-race survey was completed when most athletes are at peak training volume. Thus overtraining, which causes mood disturbance, may have skewed the results of the pre-race survey.

Future studies may yield different findings if post-race measures of depression are completed sooner post-race. Participants were advised to complete the post-race inventory and BDI-II 2 weeks post-race, as the BDI-II instructs respondents to consider how they have felt over the past 2 weeks when responding to items. Declines in mood post-race may be common and notable but short-lived for most individuals. If individuals do indeed experience a brief decline in mood postrace, a measure completed sooner post-race may capture this phenomenon better. More precise description of post-race depression, if it exists, could be identified by more frequent administration of self-report tools.

It is possible that another self-report tool, such as the Profile of Mood States (POMS), should be used in future studies - particularly if post-race dysphoria is a mood state other than depression. The POMS has been used extensively in athletic or exercise samples - 258 published articles from 1971 to $1998 .^{16}$ It is a self-report measure designed to measure six dimensions of mood: tension-anxiety, depressiondejection, anger-hostility, vigour-activity, fatigue-inertia, and confusion-bewilderment. The POMS consists of 65 adjectives describing feeling and mood which are answered on a fivepoint Likert-type scale ranging from 'not at all' to 'extremely'.

A vast body of sports psychology literature has implicated running in a variety of psychopathological conditions including but not limited to anorexia, bulimia, and obsessive-compulsive behaviour.? A tool designed to examine addiction may be considered as an additional depression measure in future studies examining post-race dysphoria. ${ }^{27}$ A body of literature is evolving that suggests that exercise deprivation; as experienced during post-race recovery when triathletes dramatically decrease training volume and intensity, can result in a state of self-perceived loss of wellbeing..$^{30}$ The aetiology is likely physiological and psychological. In a study of 40 habitual male runners, Morris and colleagues suggest that exercise may be addictive, and go on to describe a withdrawal syndrome characterised by depressive symptoms in those runners forced to stop exercising. ${ }^{17,20}$ Future research employing an addiction inventory in a population of triathletes whose post-race exercise is closely monitored may offer further understanding in this regard.

Exercise is psychoactive. Running increases positive mood and decreases negative mood. ${ }^{20.21}$ Exercise is prescribed by health professionals to treat a variety of physiological and psychological maladies. ${ }^{29}$ In addition to elevating 
mood, exercise may attenuate withdrawal symptoms during smoking cessation. ${ }^{3}$ Individuals self-medicate their depressive symptoms with substances such as nicotine, alcohol, and cannabis. ${ }^{4,16,25}$ We did not ask triathletes in our study about preand post-race mood-altering substance use. It is possible that subjects self-medicated post-race depression or exercise withdrawal with alcohol or drugs.

Future studies should thoroughly catalogue the incidence and prevalence of psychiatric disease in this population of highly motivated athletes. In order to properly treat and counsel participants in endurance sports, sports physicians and psychologists should further investigate the complex interplay between extreme exertion, mood disorders, addictive behavior, and exercise withdrawal.

\section{Conclusions}

This prospective pilot study is the first to examine the effect of participation in an ultra-distance triathlon on self-reported mood. It failed to identify 'post-Ironman depression' in the majority of athletes but raises many relevant questions about the psychological characteristics of triathletes.

\section{Acknowledgements}

We would like to acknowledge the contribution of our administrative assistant, Shari Pachinger, to this project. This study was funded internally.

\section{References}

1. American Psychiatric Association. Diagnostic and Statistical Manual of Mental Disorders. 4th ed. Washington, DC: APA, 1994.

2. Beck AT, Steer RA, Brown GK. Manual for the Beck Depression Inventory 2nd ed. San Antonio, Texas: The Psychological Corporation, 1996.

3. Bock BC, Marcus BH, King TK, Borrelli B, Roberts MR. Exercise effects on withdrawal and mood among women attempting smoking cessation. Addict Behav 1999; 24: 399-410.

4. Bovasso GB. Cannabis use as a risk factor for depressive symptoms. Am Psychiatry 2001; 158: 2033-7.

5. Extri.com Real Triathlon Calendar. Available at www.xtri.com. (Last accessed 15 July 2003.)

6. Hassmen $\mathrm{P}$, Blomstrand $\mathrm{E}$. Mood change and marathon running: a pilot study using a Swedish version of the POMS test. Scand J Psychol 1991; 32 225-32S.

7. Hauck ER, Blumenthal JA. Obsessive and compulsive traits in athletes. Sports Med 2000; 14: 215-27.

8. HFP Racing Message Board. June 15, 2001. Available at www.hfpracing.com

9. Ironman USA Forum. June 15, 2001. Available at www.ironmanusa.com

10. Joesting J. Affective changes before, during, and after a 50 mile run. Percept Mot Skills 1981; 52:162.

11. Kirkby RJ. Ultraendurance running: a case study. International Journal of
Sports Psychology 1996; 27: 109-16

12. Kohl HW, LaPorte RE, Blair SN. Physical activity and cancer: An epidemiological perspective. Sports Med 1988; 6: 222-37.

13. Lane A. Relationships between perceptions of performance expectations and mood among distance runners: the moderating effect of depressed mood. J Sci Med Sport 2001; 4:116-28.

14. Leon AS, Connett J, Jacobs DR. Leisure time physical activity levels and risk of coronary heart disease and death: The multiple risk factor intervention trial. JAMA 1987; 258: 2388-95.

15. Lerman $C$, Caporaso N, Main D, Audrain J, Boyd NR, Bowman ED. Depression and self-medication with nicotine: the modifying influence of the dopamine D4 receptor gene. Health Psychol 1998; 17(1): 56-62.

16. LeUnes A, Burger J. Profile of mood states research in sport and exercise psychology: Past, present, and future. Journal of Applied Sport Psychology 2000; 12(1): 5-15.

17. Morris $M$, Salmon P. Qualitative and quantitative effects of running on mood. $J$ Sports Med Phys Fitness 1994; 34(3):284-91.

18. Morgan WP, Brown DR, Raglin JS. Psychological monitoring of overtraining and staleness. Br J Sports Med 1987; 3:107-14

19. Morgan WP, Brown DR, Raglin JS, O'Connor PJ, Ellickson KA. Personality structure, mood states, and performance in elite male distance runners. In $J$ Sport Psychol 1988; 19: 247-63.

20. Morris $M$, Steinberg $H$, Sykes EA, Salmon $P$. Effects of temporary withdraw al from regular running. J Psychosom Res 1990; 34: 493-500.

21. North TC, McCullagh P, Tran ZV. Effect of exercise on depression. Exerc Sport Sci Rev 1990; 18: 379-415

22. Paluska SA, Schwenk TL. Physical activity and mental health: Current concepts. Sports Med 2000; 3:167-80.

23. Pierce EF, Rohaley KA, Fritchley B. Sex differences on exercise dependence for men and women in a marathon road race. Percept Mot Skills 1977; 84: 991-4.

24. Regier DA, Narrow WE, Rae DS, Manderscheid RW, Locke BZ, Goodwin FK. The de facto mental and addictive disorders service system Epidemiologic Catchment Area prospective 1-year prevalence rates of disorders and services. Arch Gen Psychiatry 1993; 50(2): 85-94.

25. Rommelspacher $H$, Dufeu $P$, Schmidt LG. Harman and nonharman in alcoholism: correlations with psychopathology and long-term changes. Alcoho Clin Exp Res 1996;20(1):3-8

26. Schomer $\mathrm{HH}$, Dunne TT. Emotional transitions of novice runners during a 7 month marathon training programme. International Journal of Sports Psychology 1994; 25:176-86.

27. Schwarz, G. Estimating the dimension of a model. Annals of Statistics 1978 6: $461-4$.

28. Scott D. Ironman recovery (Inside Triathlon.com web site). October 31 2001. Available at www.insidetri.com/scott/article/633.2html

29. Scully D, Kremer J, Meade MM, Graham R, Dudgeon K. Physical exercise and psychological well being: a critical review. $B r J$ Sports Med 1998 32(2):111-120.

30. Szabo A. The impact of exercise deprivation on well-being of habitual exercisers. Australian Journal of Science and Medicine in Sport 1995; 27(3):68 75 .

31. Tharion WJ, Strowman SR, Rauch TM. Profile and changes in moods of ultramarathoners. Journal of Sports and Exercise Psychology 1988;10 22.9-35.

32. Triathletes of the Dead Runners Society listerve. June 15, 2001. Available at www.tri-drs.org

33. USA Triathlon Calender. Available at www.usatriathlon.org (Last accessed 15 July 2003.) 